\title{
Mejoramiento de la calidad del café soluble utilizando el método Taguchi
}

\author{
Improving the quality of soluble coffee using the Taguchi method \\ Amparo Zapata Gómez ${ }^{1} \quad$ William Ariel Sarache Castro ${ }^{1}$ \\ Recibido 20 de junio de 2012, aceptado 9 de septiembre de 2013 \\ Received: June 20, 2012 Accepted: September 9, 2013
}

\begin{abstract}
RESUMEN
Con el fin de mejorar la calidad de los productos es necesario el mejoramiento de los procesos. Esto se puede lograr mediante la realización de experimentos que permitan establecer las características de calidad e implantar condiciones de operación bajo control. En la elaboración de café soluble, el método Taguchi no ha sido utilizado de forma generalizada, es posible por la complejidad del mismo y por las particulares del proceso. Por tanto, el objetivo del presente artículo es exponer los resultados obtenidos de la aplicación de un procedimiento basado en el método Taguchi, en una empresa productora de café soluble. El método facilitó la experimentación con factores no controlables, la depuración de las variables del proceso de producción de café soluble que afectaban sus resultados y la evaluación cuantitativa de la pérdida de calidad debido a variaciones funcionales. En forma adicional, los resultados ofrecen información valiosa para las empresas del sector, que les permite abordar proyectos de mejoramiento de la calidad en este tipo de procesos.
\end{abstract}

Palabras clave: Café soluble, calidad, diseño experimental, método Taguchi, mejora continua.

\begin{abstract}
In order to enhance the quality of products, the improvement of processes is required. This may be accomplished by means of experiments to establish the characteristics of quality and required conditions to keep the operation under control. In the elaboration of soluble coffee, the Taguchi Method has not been used widely, possibly due to its complexity and special features of the process. Therefore, the aim of this paper is to show the results obtained from a procedure that was applied based on Taguchi Method, in a manufacturing company of soluble coffee. The method allowed for the experimentation with uncontrollable factors, the depuration of the variables of the production process that affected their results and the quantitative evaluation of the quality loss due to functional variations. Furthermore, the results provide valuable information for companies in the sector, allowing them to undertake projects to improve the quality of this type of processes.
\end{abstract}

Keywords: Soluble coffee, quality, experimental design, Taguchi method, continuous improvement.

\section{INTRODUCCIÓN}

Se denomina café a la bebida preparada a partir de las semillas procesadas del fruto de los cafetos; este se caracteriza por un agradable aroma y sabor debido a sus propiedades organolépticas [1]. Su atractivo está muy ligado al placer de degustar una taza de café y al concepto generalizado de su carácter estimulante y energizante [2]. El café soluble es el extracto de café obtenido a partir de la semilla tostada y molida y se reconoce por sus rasgos diferenciadores en el sabor, aroma, olor y apariencia física.

1 Departamento Ingeniería Industrial. Universidad Nacional de Colombia, Sede Manizales. Manizales, Colombia. E-mail: azapatago@unal.edu.co; wasarachec@unal.edu.co 
Las características más apreciadas relacionadas con la calidad son el buqué (aroma), la acidez, el cuerpo y el sabor. La calidad del café soluble se puede juzgar con la evaluación de las características físicas y organolépticas del grano que, de forma metódica y consistente, identifican los sabores y aromas más característicos, al igual que los sabores desagradables y no característicos. La evaluación sensorial permite la caracterización de un lote de café. La prueba de taza es la valoración de las particularidades del café realizada por los catadores, buscando reconocer sus cualidades y defectos.

Existen diversos problemas en la industria del café soluble relacionados con la dificultad para controlar los parámetros físicos, químicos, microbiológicos, organolépticos y estéticos. Otro problema es el fenómeno de depósito de fluoruros a lo largo de la línea de producción [3]. Este fenómeno es causado por efectos de la floculación que se presenta de naturaleza termodinámica que obedece a cambios de temperatura, presión, composición del fluido de la taza y puede ser muy difícil de resolubilizar [3]. De modo adicional, el control de humedad es un factor de alto impacto en la calidad de la taza de café, con el fin de evitar la proliferación de microorganismos y la actividad enzimática.

Dicha situación exige establecer un sistema de control orientado a mantener la temperatura y el tiempo de procesamiento adecuados que permitan alcanzar los niveles deseados en la composición de cafeína, hidratos de carbono, proteína y minerales [3]. Un inadecuado manejo del proceso, debido a la sensibilidad del producto, puede dar como resultado una taza de café con diferentes componentes aromáticos, con variaciones en el color y densidad. Bajo esta situación, el método Taguchi se expone como una herramienta útil dada su capacidad para analizar diferentes variables simultáneamente mediante el desarrollo de experimentos que agilizan las soluciones e interpretación de los datos.

El método Taguchi ha sido bastante aplicado en la mejora de procesos y productos en diversos sectores como la industria de alimentos [4-7], farmacéutica [8], electrónica [9-10], vidrios y envases [11-12], aeronáutica [13], hidrocarburos [14], software [15] y en materiales de construcción [16-17], entre otros; sin embargo, en la literatura consultada no fueron encontrados antecedentes de aplicaciones del método en el sector objeto de estudio.

En este sentido, la presente contribución expone una aplicación del método Taguchi para el análisis de las variables del proceso de producción de café soluble con el fin de mejorar su calidad y estandarizar la operación para mantenerla controlada y estable. Aunque la aplicación se realizó en una empresa piloto de la industria cafetera colombiana, el procedimiento expuesto puede ser aplicado, con los debidos ajustes, en otras empresas del sector o empresas del sector de alimentos con problemas similares.

\section{ANÁLISIS TEÓRICO}

Como elemento de partida, se reconoce que "...el término calidad total es quizás el que se ha utilizado más para denominar a esta nueva corriente de Taguchi, que en sintesis se puede resumir cómo hacer las cosas bien en todas las actividades de la empresa para satisfacer las necesidades de los consumidores o clientes" [5]. Más allá de cumplir con las especificaciones de un producto, Taguchi entiende la calidad como el resultado de un proceso de producción estable, con mínimas variaciones y capaz de generar productos de mayor confiabilidad, mejor funcionamiento, menores costos y menores tiempos de diseño y desarrollo [18].

El método Taguchi se expone como una herramienta eficiente para el diseño y optimización de procesos y productos, debido a que se centra en la identificación y evaluación de las variables con mayor influencia en la salida del proceso, en la reducción de los efectos de los factores no controlables y en la reducción de la variación del desempeño del proceso [19]. Otros autores identifican el Método Taguchi como una técnica que ayuda a obtener una combinación óptima de diseño de parámetros para que el producto sea funcional y con un alto nivel de calidad [20-22].

El método se basa en el estudio de los efectos principales generados por la ocurrencia de algo no controlado [19] e incorpora en su análisis las variaciones que afectan en forma negativa a la calidad, mediante el uso de experimentos, el análisis de datos, el análisis de interacciones y la comprobación [21]. El análisis se realiza sobre los efectos principales y permite definir las condiciones óptimas para asegurar el diseño del proceso o del producto [22]. 
El método Taguchi no solo se emplea en la investigación de las relaciones causales, sino que busca desarrollar un modelo matemático de causa y efecto y contribuir a la selección de los efectos principales para consolidar la robustez del diseño del producto y del proceso. Es aplicable en procesos donde sea bastante costoso el manejo de los niveles para cada uno de los factores involucrados en la experimentación y, aunque existen otros métodos aplicables [23], ofrece ventajas superiores como: mayor poder de estimación de las interacciones, el tamaño de los arreglos experimentales, la efectividad de las soluciones, la facilidad de su aplicación, la buena relación beneficio/costo, la flexibilidad y la efectividad de los métodos propuestos para el análisis de los resultados [24-27].

Desde el punto de vista metodológico, el método Taguchi se sustenta en el concepto de aleatorización y análisis de varianza para asegurar productos robustos, de alta calidad y de bajo costo. Para tal fin, se apoya en una secuencia de pasos para asegurar la obtención de los datos apropiados, de modo que permitan un análisis objetivo que conduzca a deducciones válidas respecto del problema establecido. La expresión 1 expone el modelo sobre el cual se sustenta el método.

$$
\begin{gathered}
\gamma=\alpha_{0}+\alpha_{1} x_{1}+\alpha_{2} x_{2}+\alpha_{3} x_{3}+\cdots \cdot . \\
+\alpha_{11} x_{1}+\alpha_{12} x_{1} x_{2}+\alpha_{23} x_{2} x_{3}+ \\
\cdots . .+\alpha_{i j} x_{i} x_{j}
\end{gathered}
$$

Donde:

$\alpha_{i}=$ Coeficiente de regresión del efecto principal del factor $i$.

$\alpha_{i j}=$ Coeficiente de regresión de la interacción entre los factores $i$ y $j$.

$x_{i}=$ Factores controlables del proceso.

$\gamma=$ Variable dependiente.

Lo particular del método son los arreglos ortogonales, los cuales son factoriales fraccionados para la realización del experimento, así como la utilización de una medida de variabilidad para la realización del análisis de resultados. Este método permite hacer una evaluación matemática e independiente del efecto de cada uno de los factores presentes en el diseño.

\section{Descripción del proceso}

Para comprender la dinámica del análisis se parte de reconocer el café como la semilla del fruto de la planta del género Coffea, descascarado, con cafeína y listo para el tostado de acuerdo con las especificaciones en la norma de café verde y las especificaciones y métodos de prueba de las Normas ISO [28-32]. La Figura 1 expone la secuencia general del proceso de producción de café soluble, el cual corresponde a una configuración en línea orientada a la separación y concentración de los sólidos solubles de café, hasta convertirlos en un polvo fino.

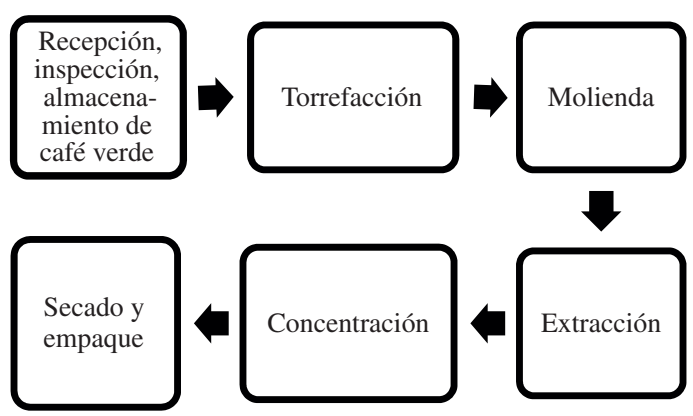

Figura 1. Diagrama del proceso de café soluble.

Una descripción general de las operaciones es la siguiente:

\section{Recepción, inspección y almacenamiento de café} verde: el café que llega a la planta es sometido a un análisis de calidad para determinar si se encuentra dentro de las especificaciones necesarias para su procesamiento; si este es aprobado, es almacenado temporalmente en silos.

Torrefacción: el café se expone a calentamiento para liberar la humedad del grano, se realiza en movimiento continuo de forma tal que se garantice la homogeneidad de todas las partículas. Durante el tostado ocurre un proceso de descomposición térmica que genera cambios químicos y físicos que desarrollan el sabor, color y aroma característicos del producto final. La torrefacción se realiza en tres fases: "fase de secado (proceso endotérmico), fase de tueste o pirólisis (reacciones pirolíticas de carácter exotérmico dentro de la célula) y fase de enfriamiento (enfriamiento por aire y el agua)"[33].

Molienda: en la molienda hay una reducción de tamaño del grano buscando incrementar la 
superficie de contacto de manera que se favorezca la transferencia de sólidos solubles en la etapa de extracción.

Extracción: parte del café tostado y molido se disuelve en el agua y pasa a ser parte del líquido en forma de sólidos solubles. Como actividad complementaria se realiza la filtración, enfriamiento, clarificación y almacenamiento temporal del extracto diluido.

Concentración: consiste en reducir el contenido de agua en el extracto de café, el sistema más utilizado es la evaporación realizada al vacío. Se usan intercambiadores de calor donde se busca conservar las propiedades del café, calentándolo el menor tiempo posible y en forma muy homogénea. El resultado de la concentración se somete a otro almacenamiento temporal del extracto concentrado.

Secado y empaque: el secado del producto se hace en una torre de deshidratación en la que se inyecta el café ya extraído en forma de una ducha muy fina. El producto final se envasa en forma habitual en jarras de vidrio y es cerrado de manera hermética para evitar la entrada de oxígeno y humedad al producto.

\section{METODOLOGÍA}

El procedimiento utilizado para abordar el problema se expone en la Figura 2. Para su aplicación, se seleccionó una empresa de producción de café soluble ubicada en la zona cafetera colombiana. En ella se muestran los pasos realizados para la aplicación del método.

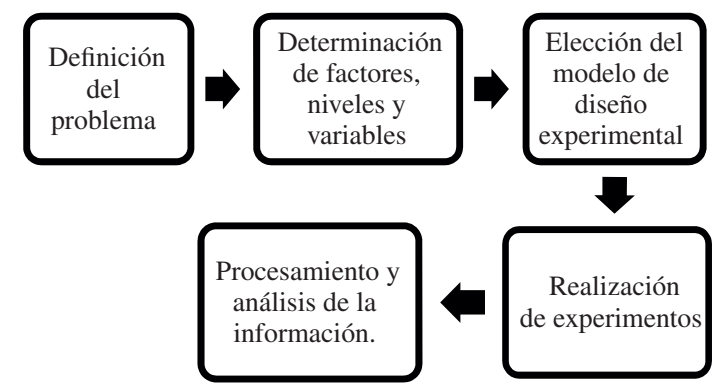

Figura 2. Desarrollo del diseño de experimentos.

Definición del problema: El problema radica en que debido a la complejidad del proceso existe un número alto de factores influyentes que dificultan la estandarización de las operaciones y exigen un proceso experimental que puede resultar costoso. Asimismo, investigaciones previas demostraron que las interacciones entre los factores principales son débiles [34].

Determinación de factores, niveles y variables: para el caso en estudio, el trabajo conjunto de la empresa y los investigadores llevó a obtener una mejor planeación del experimento y a definir las siguientes variables para el análisis:
A: Tipo de secado del café en grano.
B: Tiempo de la extracción de sólidos solubles.
C: Temperatura de la extracción de sólidos solubles.
D: Tiempo de concentración.
E: Tiempo de secado del extracto de café concentrado.
F: Tamaño del grano.
G: Relación de aroma/buqué.

En esta etapa es importante realizar un breve estudio de las variables seleccionadas. Se realizó una primera fase experimental, en la cual se analizaron tres variables como variables ruido porque se dudaba de la influencia en la extracción; estas variables fueron A, C y F. De este primer análisis se concluyó que la variable ruido más significativa era el tipo de secado del café en grano al momento de ser alimentado el tándem de extracción. En la segunda fase experimental se involucraron para el ajuste las variables $\mathrm{B}, \mathrm{D}$ y $\mathrm{E}$.

\section{Elección del modelo de diseño experimental:}

El modelo se justifica debido al cambio en las condiciones de operación del proceso y por la necesidad de medir el efecto sobre una o varias propiedades. Igualmente, por el concepto de interacción, es decir, el efecto que un factor de entrada "A" tiene sobre el efecto de otro factor de entrada "B". En otras palabras, el efecto que "B" tiene sobre la variable de respuesta depende del estado en el cual se encuentra "A".

Para el diseño del experimento, el número de réplicas se determinó utilizando la expresión 2:

$$
r=\left[\left(Z_{\alpha / 2}+Z_{\beta}\right)^{2} \alpha^{2}\right] / \delta^{2}
$$

Donde:

$\alpha=$ Probabilidad de error tipo I.

$\beta=$ Probabilidad de error tipo II. 
$\delta=$ Diferencia en los resultados.

$Z=$ Valor tipificado de la distribución Normal estándar.

$\sigma=$ Desviación estándar.

Así se obtiene que:

$$
\begin{gathered}
Z_{\alpha / 2}=1,95 ; \quad Z_{\beta}=1,65 ; \quad \delta=2,4 \sigma \\
\text { y } r=2,2495
\end{gathered}
$$

Basados en las pruebas experimentales realizadas y en el conocimiento y experiencia en el proceso, se definieron los siguientes niveles para cada una de las variables de control según se muestra en la Tabla 1.

Tabla 1. Niveles para las variables de control.

\begin{tabular}{|c|l|l|}
\hline Variable & \multicolumn{1}{|c|}{ Nivel I } & \multicolumn{1}{c|}{ Nivel II } \\
\hline A & $\begin{array}{l}\text { Secado en condiciones } \\
\text { ambientales } 18^{\circ} \mathrm{C} \\
(24 \mathrm{hrs} .)\end{array}$ & $\begin{array}{l}\text { Secado calentador } \\
50^{\circ} \mathrm{C}(4 \mathrm{hrs} .)\end{array}$ \\
\hline B & $1 \mathrm{hr}$. & $2 \mathrm{hrs}$. \\
\hline C & $40^{\circ} \mathrm{C}$ & $50^{\circ} \mathrm{C}$ \\
\hline D & $16 \mathrm{hrs}$. & $24 \mathrm{hrs}$. \\
\hline E & 30 & 60 \\
\hline F & Mallas $17 \mathrm{y} 18$. & Mallas $20 \mathrm{y} 24$. \\
\hline G & $125\left(\mathrm{ouE} / \mathrm{m}^{3}\right)$ & $250\left(\mathrm{ouE} / \mathrm{m}^{3}\right)$ \\
\hline
\end{tabular}

En la Tabla 1 cada variable a dos niveles tiene (2-1) $=1$ grados de libertad (gl). Se estableció que no había interacciones entre los efectos principales. Por tanto:

Total $\mathrm{gl}=(7$ variables $\mathrm{x} 1 \mathrm{gl})+(0$ interacciones $\mathrm{x}$ 1gl.) $=7 \mathrm{gl}$.

Taguchi [35] definió uno de los arreglos ortogonales como el $\mathrm{L}_{8}(8$ corridas en un diseño de 7 factores a dos niveles cada uno); dado que el diseño experimental cumple con los dos requisitos, se consideró apropiado para este caso.

Realización de experimentos: Los resultados de la experimentación bajo un diseño $\mathrm{L}_{8}$ se muestran en la Tabla 2 con sus respectivas réplicas y promedios. En ella se exponen los resultados de los arreglos ortogonales tomando como variable de respuesta la relación aroma/buqué involucrada en la experimentación; por su parte, el tipo de secado del café en grano se asumió como variable ruido, según lo encontrado en la anterior fase de experimentación.
Tabla 2. Resultados de los arreglos ortogonales $\mathrm{L}_{8}$.

\begin{tabular}{|c|c|c|c|c|c|c|c|c|c|}
\hline $\mathbf{N}^{\mathbf{0}}$ & $\mathbf{A}$ & $\mathbf{B}$ & $\mathbf{C}$ & $\mathbf{D}$ & $\mathbf{E}$ & $\mathbf{F}$ & $\mathbf{G}$ & \multicolumn{2}{|c|}{ Réplicas promedio } \\
\hline $\mathbf{1}$ & $\mathbf{1}$ & $\mathbf{2}$ & $\mathbf{3}$ & $\mathbf{4}$ & $\mathbf{5}$ & $\mathbf{6}$ & $\mathbf{7}$ & $\mathbf{1}$ & $\mathbf{2}$ \\
\hline $\mathbf{2}$ & 1 & 1 & 1 & 1 & 1 & 1 & $\begin{array}{c}50,00 \\
51,10\end{array}$ & 54,25 \\
\hline $\mathbf{3}$ & 1 & 2 & 2 & 1 & 1 & 2 & 2 & $\begin{array}{c}38,00 \\
39,75\end{array}$ & 40,00 \\
\hline $\mathbf{4}$ & 1 & 2 & 2 & 2 & 2 & 1 & 1 & $\begin{array}{c}37,00 \\
38,33\end{array}$ & 40,66 \\
\hline $\mathbf{5}$ & 2 & 1 & 2 & 1 & 2 & 1 & 2 & $\begin{array}{c}56,74 \\
57,00\end{array}$ & 59,50 \\
\hline $\mathbf{6}$ & 2 & 1 & 2 & 2 & 1 & 2 & 1 & $\begin{array}{c}58,45 \\
59.00\end{array}$ & 60,00 \\
\hline $\mathbf{7}$ & 2 & 2 & 1 & 1 & 2 & 2 & 1 & $\begin{array}{c}45,80 \\
39,65\end{array}$ & 43,50 \\
\hline $\mathbf{8}$ & 2 & 2 & 1 & 2 & 1 & 1 & 2 & $\begin{array}{c}52,50 \\
51,75\end{array}$ & 51,00 \\
\hline
\end{tabular}

Cada experimento ofrece un resultado bajo un conjunto de condiciones desiguales, lo cual permite hacer comparaciones de los diferentes niveles de los factores. El diseño ortogonal accede a comparar los niveles de las variables bajo condiciones disímiles de la manera más eficiente. Se seleccionó un arreglo ortogonal $\mathrm{L}_{8}$ para la matriz interna, permitiendo estudiar las variables a dos niveles cada uno; asimismo, se usó la técnica del falso nivel para estudiar las variables del arreglo compuesto en la matriz externa [36].

Procesamiento y análisis de la información: para obtener la característica de calidad del producto (respuesta Y) es necesario contemplar el porcentaje de remoción correspondiente al volumen de filtración de compuestos incoloros contenidos en el agua potable. La experimentación permitió determinar el resultado de remoción del diseño así:

- $\mathrm{L}_{8}=\left(2^{7}\right)$.

- $\quad$ Número de datos $=16$.

- Suma total de los datos $\mathrm{T}=398,93$ y $\overline{\mathrm{T}}=49,50$.

Este factor es el que se debe controlar y considerar para optimizar la taza de café. El cálculo de la respuesta promedio para cada nivel es el siguiente:

$\overline{\mathrm{A}}_{1}=(51,10+48,15+39,75+38,33) / 4$

$\overline{\mathrm{A}}_{1}=44,3325$.

$\overline{\mathrm{A}}_{2}=(57,00+59,00+39,65+51,75) / 4=51,85$

El cálculo del efecto principal de cada variable es el siguiente: 
$\overline{\mathrm{A}}=$ Efecto principal de $\mathrm{A}=\overline{\mathrm{A}}_{1}-\overline{\mathrm{A}}_{2}$

$\overline{\mathrm{A}}=(44,33-51,85)=-8,52$.

El resultado indica la variación promedio de las respuestas cuando se cambia de factor. Para interpretar el efecto principal se comprobó que la variación observada en la respuesta es debida a un efecto real de cada factor planteado [37]. De igual forma se calcularon los efectos para cada variable. La Tabla 3 muestra los resultados de la respuesta de los efectos principales.

Tabla 3. Efectos principales.

\begin{tabular}{|l|c|c|c|c|c|c|c|}
\hline Nivel & A & B & C & D & E & F & G \\
\hline Nivel 1 & 44,33 & 54,58 & 48,33 & 47,95 & 50,84 & 50,50 & 47,99 \\
\hline Nivel 2 & 51,85 & 40,02 & 49,12 & 50,00 & 46,60 & 46,90 & 49,49 \\
\hline Nivel (1-2) & $-8,52$ & 14,56 & $-0,79$ & $-2,05$ & 4,24 & 3,60 & $-1,50$ \\
\hline
\end{tabular}

En la Figura 3 se grafican los resultados de la Tabla 3 para exponer el comportamiento de las variables. En esta se muestran las relaciones entre las pendientes de las líneas y su correspondencia con el efecto de la variable sobre la respuesta.

Average Etaby Factor Levels (Dashed line indicates $\pm 2 *$ Standar Error

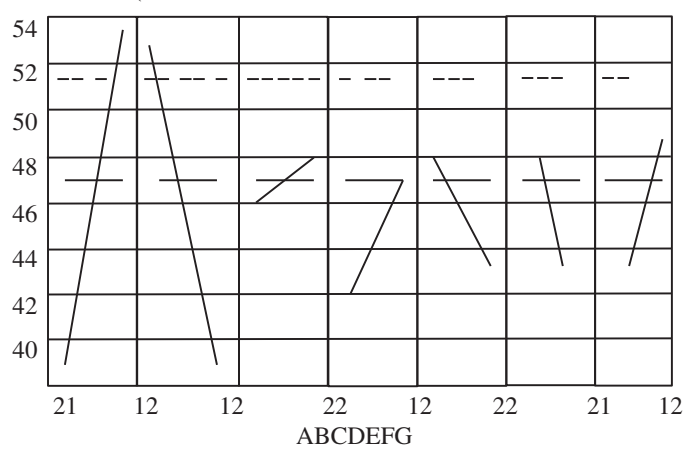

Figura 3. Efectos factoriales.

Así, mientras mayor sea la pendiente, más fuerte es el efecto de la variable [38]. Este es un diseño con columnas con solo efectos principales y sin efectos de interacción. De acuerdo con los resultados, los mejores niveles de operación del sistema son $\mathbf{A}_{\mathbf{2}} \mathbf{B}_{\mathbf{1}} \mathbf{C}_{\mathbf{2}} \mathbf{D}_{\mathbf{2}} \mathbf{E}_{\mathbf{1}} \mathbf{F}_{\mathbf{1}} \mathbf{G}_{\mathbf{2}}$, los cuales muestran las mejores relaciones señal-ruido. Al sustituir el nivel 2 por el nivel 1 se obtuvo la combinación $\mathbf{A}_{\mathbf{2}} \mathbf{B}_{\mathbf{1}} \mathbf{C}_{2} \mathbf{D}_{\mathbf{2}} \mathbf{E}_{\mathbf{1}} \mathbf{F}_{\mathbf{1}} \mathbf{G}_{\mathbf{2}}$, donde se optimiza todo tipo de recursos, se balancea la línea de producción y por consiguiente se estandarizan las operaciones. Como este resultado fue mayor que la media estimada y calculada al comienzo, entonces hay una alta probabilidad de reproducir los resultados [39].

Con el objetivo de ratificar los resultados se debe generar una estimación de la respuesta (porcentaje de remoción) y compararlos con los resultados del experimento realizado y validado. Para el cálculo de la estimación de la media del proceso se tuvieron en cuenta de modo estricto los efectos fuertes, para no obtener una sobreestimación o no confundir el error experimental con los promedios, así:

$$
\begin{gathered}
\mu=\bar{T}+\left(\bar{A}_{2}-\bar{T}\right)+\left(\bar{B}_{2}-\bar{T}\right)+ \\
\left(\bar{C}_{2}-\bar{T}\right)+\left(\bar{D}_{2}-\bar{T}\right)+\left(\bar{E}_{2}-\bar{T}\right)+ \\
\left(\bar{F}_{1}-\bar{T}\right)+\left(\bar{G}_{2}-\bar{T}\right)
\end{gathered}
$$

Donde:

$\overline{\mathrm{T}}$ : Media global.

$\overline{\mathrm{A}}_{2}, \overline{\mathrm{B}}_{2}, \overline{\mathrm{C}}_{2}, \overline{\mathrm{D}}_{2}, \overline{\mathrm{E}}_{2}, \overline{\mathrm{F}}_{1}$ y $\overline{\mathrm{G}}_{2}$ : Efectos promedio de cada factor.

$\mu=64,021$, considerando todos los efectos.

$\mu=64,815$, omitiendo solo el efecto más pequeño.

Al recalcular la media del proceso sin considerar las variables nulas y poco significativas, se encontró que $\mu=61,99$, por lo cual se concluye que el modelo obtenido solo capta un porcentaje pequeño de la variabilidad total, dejando el restante a la influencia del ruido presente en la fase experimental. Esto se debe a la exclusión de las interacciones entre los factores experimentales [40].

\section{CONCLUSIONES}

Los resultados obtenidos permitieron mejorar las características de calidad de la taza de café y aportaron al grupo directivo información valiosa para la toma de decisiones relacionada con el mejoramiento de sus productos. Con la aplicación del método Taguchi se pudieron analizar los factores controlables y no controlables y se identificaron los aspectos críticos del proceso que permiten la iniciación de acciones de mejoramiento continuo.

El análisis de regresión y varianza permitió demostrar que, de los siete factores estudiados, los que influyen significativamente en la calidad de la taza de café fueron la concentración y el aroma en su máximo 
nivel de desempeño deseado, lo mismo sucedió con el extracto de café concentrado; mientras que la variable tamaño del café en grano lo hizo en su mínimo nivel de especificaciones. En forma conjunta estas variables independientes tienen un alto porcentaje de participación en la calidad de la taza de café.

De la primera fase experimental se dedujo que las tres variables ruido que tenían gran influencia en la extracción fueron el tipo de secado, la temperatura de la extracción y el tamaño del grano de café. De este primer análisis se concluyó que la variable ruido más significativa era el tipo de secado al momento de ser alimentado el tándem de extracción.

La experimentación permitió determinar que el factor que se debe controlar y considerar para optimizar la taza de café corresponde al volumen de filtración de compuestos incoloros contenidos en el agua potable; asimismo, los resultados permiten concluir que el efecto de la extracción en la variabilidad de la respuesta es en la práctica nulo y que el efecto de las variables temperatura, concentración y aroma no son significativos según se demuestra con el análisis de varianza.

Conocidas las variables que influyen de manera importante en las características de la taza de café, se anexaron los factores no significativos de tipo de secado del café en grano y tamaño del grano ambos en sus niveles mínimos, como la mejor combinación de las variables seleccionadas en este estudio, la que fue validada con posterioridad con un ensayo experimental confirmatorio. En cuanto a la variación de las características de la taza de café durante el envase, no se detectaron diferencias significativas tanto en el sabor como en la acidez del producto. La taza de café así optimizada constituye una buena alternativa para este tipo de producto, debido a la presencia de un muy buen aroma y sabor.

Para el caso de estudio, dadas las particularidades del proceso de producción, la aplicación del método Taguchi permitió detectar el conjunto de variables críticas a partir de una valoración integral de su desempeño, en función de los criterios más relevantes para la empresa. Asimismo, permitió cuantificar el efecto de cada variable de entrada sobre la variable de respuesta y sus interacciones. $\mathrm{La}$ metodología aplicada permitió llegar a conclusiones válidas, disminuyendo el número de ensayos, lo cual repercute en forma favorable en los costos del proceso experimental.

\section{REFERENCIAS}

[1] M. Gotteland y P. Saturnino. "Algunas verdades sobre el café". Revista Chilena de Nutrición. Vol. 34 No 2, pp. 105-115. Junio 2007. ISSN: 0717-7518.

[2] A. Valenzuela. "El café y sus efectos en la salud cardiovascular y en la salud materna". Revista Chilena de Nutrición. Vol. 37 $\mathrm{N}^{\circ}$ 4, pp. 514-523. Diciembre 2010. ISSN: $0717-7518$.

[3] A. Zapata. "Problemas típicos de calidad en la industria del café". Informe de investigación. Doctorado en ingeniería. Universidad Nacional de Colombia, Sede Manizales. Colombia. Junio 2011.

[4] P. Medina, E. Cruz y J. Restrepo. "Aplicación del modelo de experimentación Taguchi en un ingenio azucarero del Valle del Cauca". Revista Scientia et Technica, año XIII 13 $\mathrm{N}^{\circ}$ 34, pp. 337-341. Mayo 2007. ISSN: 0122-1701.

[5] P. Chuaqui, E. Wittig de Penna and M. Villarroel. "Método de Taguchi para optimizar calidad de postres funcionales destinados al adulto mayor y estudio de prefactibilidad técnico - económica”. Revista Chilena de Nutrición. Vol. 31 N$^{\circ}$ 2, pp. 118-127. Agosto 2004. ISSN: 0717-7518.

[6] M. Villarroel, J. Hazbun y P. Morales. "Desarrollo de una formulación de paté a base de descartes de pulpa de trucha arcoíris". Revista Archivo Latinoamericano de Nutrición. Vol. 60 No 2, pp. 199-204. Junio 2010. ISSN: 0004-0622.

[7] M. Villarroel, E. Uquiche, J. Urbulú. "Caracterización sensorial de paté a base de descartes de pulpa de salmón utilizando la metodología superficie de respuesta". Archivo Latinoamericano de Nutrición. Vol. 49 N$^{\circ}$ 3, pp. 265-270. Septiembre 1999. ISSN: 0004-0622.

[8] E. Yacuzzi, F. Martin, H. Quiñones y M. Popovsky. "El diseño experimental y los métodos Taguchi: conceptos y aplicaciones en la industria farmacéutica". Serie documentos de trabajo Universidad del CEMA, pp. 1-30. 
Febrero 2004. Fecha de consulta: 10 de mayo de 2010. URL: http://www.ucema.edu.ar/ publicaciones/download/documentos/258. pdf

[9] N. Xydas, D. Tsi, V. Gurevich, M. Krichever and I. Kao. "Dynamic Taguchi Methods and Parameter Design as Applied in Barcode Scanning and Scanners". Concurrent Engineering: Research and Applications. Vol. 13, Issue 1, pp. 69-80. December, 2005. DOI: 10.1177/1063293X0506198.

[10] Y. Báez, J. Limón, D. Tlapal y M. Rodríguez. "Aplicación de Seis Sigma y los Métodos Taguchi para el Incremento de la Resistencia a la Prueba de Jalón de un Diodo Emisor de Luz”. Información Tecnológica. Vol. 21 $\mathrm{N}^{\mathrm{o}}$ 1, pp. 63-76. 2010. ISSN: 0718-0764. DOI:10.1612/inf.tecnol.4141it.08.

[11] R. Niebles. "A new approach to problems of multiple responses using loss functions". International Journal of Quality Management. Vol. 17, Issue 2, pp. 132-143. April, 2010. ISSN: 0591820676.

[12] D. García y V. Márquez. "La aplicación práctica del Método Taguchi en la producción de envases de plástico de 120 ml". Tesis de Licenciatura en Estadística. Universidad de Los Andes, Facultad de Ciencias Económicas y Sociales, Escuela de Estadística. Mérida, Venezuela. 1996.

[13] M. Ramiro-González "Estudio de la situación actual en España del diseño robusto y aplicación de su metodología a una empresa del sector aeronáutico a través de las herramientas VMEA y diseño de experimentos". Escuela Superior de Ingenieros de Sevilla, pp. 138-149. Fecha de consulta: agosto 16 de 2011. URL:http:// bibing.us.es/proyectos/abreproy/3847

[14] M. Hernández, S. Santillán, G. Volodymyr, M. López. "Caso de aplicación del método Taguchi en el diseño de herramientas de muestreo de Hidrocarburos". Revista Ingeniería Mecánica. Vol. 1 Nº 3, pp. 91-101. Septiembre 2003. URL: http://revistasomim. net/revistas/1_3/art3.pdf

[15] A. Pentón. "Aplicación de la Tabla Ortogonal en el diseño de los Casos de prueba de Software". Revista Avanzada Científica. Vol. $15 \mathrm{~N}^{\circ}$ 2, pp. 1-12. Junio 2012. ISSN: 1029-3450.
[16] K. Paricaguan. "Desarrollo de un recubrimiento epóxico reducible con agua". Revista Ingeniería UC. Vol. $17 \mathrm{~N}^{\mathrm{o}}$ 2, pp. 27-37. Agosto 2010. ISSN: 1316-6832.

[17] P. Ross. "Taguchi Techniques for Quality Engineering”. McGraw-Hill, pp. 279-286. New York, USA. 2012. ISBN: 0070539588.

[18] D. Montgomery. "Diseño y análisis de experimentos". Grupo Editorial Iberoamérica. Ed. 4.1, pp. 485. Madrid, España. 1999.

[19] G. Box. "Signal-to-Noise Ratios, Performance Criteria, and Transformations". Technometrics. Vol. $30 \mathrm{~N}^{\circ} 1$, pp. 1-17. February, 1998. ISSN: 0040-1706. Fecha de consulta: agosto 2010. URL: http://www. jstor.org/stable/1270311?seq=1.

[20] A. Atkinson, A. Donev and R. Tobias. "Optimum Experimental Designs, with SAS". Oxford University Press, p. 5. Paperback, Published 2010. ISBN: 978-0-19-929660-6. 2007.

[21] R. Sreenivas, C. Ganesh, S. Prakasham and P. Hobbs. "The Taguchi methodology as a statistical tool for biotechnological applications: A critical appraisal". Biotechnology Journal. $\mathrm{N}^{\circ} 3$, pp. 510-523. October 2008. ISSN: 1860-7314 DOI: 10.1002/biot.200700201.

[22] R. León, A. Shoemaker and R. Kacker. "Performance measures independent of adjustment: an explanation and extension of Taguchi's signal-to-noise ratios". Technometrics. Vol. 29, Issue 3, pp. 253287. August, 1987. ISSN: 0040-1706. DOI:10.1080/00401706.1987.10488231

[23] P. Medina, E. Cruz y C. Zapata. "Comparación de las metodologías Shainin y Taguchi del diseño experimental en un ingenio azucarero del Valle del Cauca". Revista Scientia et Technica, Año XVI No 45, pp. 245-250. Agosto 2010. ISSN: 0122-1701.

[24] N. Vijayan, B. Abrahamb, J. MacKayb, G.Boxc, R. Kackerd, T. Lorenzene, J. Lucasf, R. Myersg, G. Viningg, J. Nelderh, M. Phadkei, J. Sacksj, W. Welchj, A. Shoemakerk, K. Tsuik, S. Taguchil and J. Wua. "Taguchi's Parameter Design: A Panel Discussion”. Technometrics. Vol. 34, Issue 2, pp. 127-161. May, 1992. ISSN: 0040-1706.

[25] K. Anand. "Improving Paraffin Wax Yield Through Process Optimization Using 
Taguchi's Method of Experimentation". Quality Engineering. Vol. 6, Issue 1, pp. 39-56. April, 2007. ISSN: 0898-2112.

[26] K. Bhote. "World Class Quality Using Desing of Experiments to Make it Happen". American Management Association, pp. 110-114. New York, USA. 1991. ISBN: 0-8144-0427-8.

[27] D. Wang. "Determination of Nattokinase Production Condition Using Taguchi Parameter Desing". Food Science and Technology International. Vol. 12, Issue 3, pp. 215-220. January, 2006. ISSN: 1082-0132.

[28] International Organization for Standardization. Normas ISO 3509. "Cofee and Cofee products. Vocabulary: 2005", p. 15. Date of visit: January 29, 2010 URL: www.iso.org/ iso/catalogue_detail.htm?csnumber $=34184$

[29] International Organization for Standardization. Norma ISO 4149. "Green Cofee. Olfatory and visual examination and determination of foreign matter and defects: 2005", p. 9. Date of visit: January 19, 2010. URL: www.iso.org/ iso/catalogue_detail.htm?csnumber=35894

[30] International Organization for Standardization. Norma ISO 4150. "Green cofee or raw coffee. Size analysis. Manual and machine sieving: 2006", p. 41. Date of visit: January 2, 2010. URL:www.iso.org/iso/catalogue_detail. htm?csnumber $=44602$

[31] International Organization for Standardization. Norma ISO 6668. "Green cofee. Preparation of samples for use in sensory nalysis. 2005”, p. 4. Date of visit: January 15, 2010. URL: www.iso.org/iso/catalogue_detail. htm?csnumber $=44609$

[32] International Organization for Standardization. Norma ISO 10470. "Green cofee. Defect reference chart. 2008", p. 22. Date of visit: January 4, 2010. URL: www.iso.org/iso/ catalogue_detail.htm?csnumber $=40401$
[33] R. Clarke and R. Macrae. "Cofee: Related Beverages". Elsevier Applied Science. First Edition, pp. 94-143. London, UK. 1987. ISBN: 1-85166-103-4.

[34] A. Zapata. "Análisis de interacción de factores influyentes en la calidad de la taza de café soluble". Informe de investigación. Doctorado en ingeniería, Universidad Nacional de Colombia, Sede Manizales. Colombia. Septiembre 2011.

[35] G. Taguchi. "Introduction to quality engineering: Designing Quality Into Products and Processes". Asian Productivity Organization. Tokyo, Japan. 1988. ISBN: 9283310845.

[36] G. Taguchi and S. Konishi. "Orthogonal arrays and linear graphs: tools for quality engineering". American Supplier Institute, pp. 110-114. USA. 1998. ISBN: 094124301X.

[37] G. Taguchi. "Quality engineering in production systems". McGraw-Hill, p. 433. Windsor, USA. 1998. ISBN: 0070628300.

[38] Instituto Tecnológico de Chihuahua. "Curso de Ingeniería de la calidad". Manual del Curso online. México. Fecha de consulta: 5 de julio de 2011. URL: http://www.itch.edu. $\mathrm{mx} /$ academic/industrial/ingcalidad/index. $\mathrm{html}$

[39] N. Logothetis and H. Wynn. "Quality Through Design: Experimental Design, Off-line Quality Control, and Taguchi's Contributions". Oxford University Press, Oxford Science Publications, p. 464. Dunfermline, United Kingdom. 1989. ISBN: 0-19-851993-1.

[40] J. Cesarone. "The Power of Taguchi". IIE Solutions. Vol. 33, Issue 11, pp. 36-40. November, 2001. URL: http://www.iienet2. org/ 